\title{
Massive disk formation in the tidal disruption of a neutron star by a nearly extremal black hole
}

\author{
Geoffrey Lovelace ${ }^{1,2,3}$, Matthew D. Duez ${ }^{4}$, Francois Foucart ${ }^{5}$, \\ Lawrence E. Kidder ${ }^{1}$, Harald P. Pfeiffer ${ }^{5,6}$, Mark A. Scheel ${ }^{3}$, and \\ Béla Szilágyi ${ }^{3}$ \\ ${ }^{1}$ Center for Radiophysics and Space Research, Cornell University, Ithaca, NY, 14853, USA \\ ${ }^{2}$ Gravitational Wave Physics and Astronomy Center, California State University \\ Fullerton, Fullerton, California 92831, USA \\ 3 Theoretical Astrophysics 350-17, California Institute of Technology, Pasadena, CA \\ 91125, USA \\ ${ }^{4}$ Department of Physics \& Astronomy, Washington State University, Pullman, \\ Washington 99164, USA \\ ${ }^{5}$ Canadian Institute for Theoretical Astrophysics, University of Toronto, Toronto, Ontario \\ M5S 3H8, Canada \\ ${ }^{6}$ Fellow, Canadian Institute for Advanced Research \\ E-mail: glovelace@fullerton.edu,m.duez@wsu.edu, ffoucart@cita.utoronto.ca, \\ kidder@astro.cornell.edu, pfeiffer@cita.utoronto.ca, \\ scheel@tapir.caltech.edu, szilagyi@tapir.caltech.edu
}

\begin{abstract}
Black hole-neutron star (BHNS) binaries are important sources of gravitational waves for second-generation interferometers, and BHNS mergers are also a proposed engine for short, hard gamma-ray bursts. The behavior of both the spacetime (and thus the emitted gravitational waves) and the neutron star matter in a BHNS merger depend strongly and nonlinearly on the black hole's spin. While there is a significant possibility that astrophysical black holes could have spins that are nearly extremal (i.e. near the theoretical maximum), to date fully relativistic simulations of BHNS binaries have included black-hole spins only up to $S / M^{2}=0.9$, which corresponds to the black hole having approximately half as much rotational energy as possible, given the black hole's mass. In this paper, we present a new simulation of a BHNS binary with a mass ratio $q=3$ and black-hole spin $S / M^{2}=0.97$, the highest simulated to date. We find that the black hole's large spin leads to the most massive accretion disk and the largest tidal tail outflow of any fully relativistic BHNS simulations to date, even exceeding the results implied by extrapolating results from simulations with lower black-hole spin. The disk appears to be remarkably stable. We also find that the high black-hole spin persists until shortly before the time of merger; afterwards, both merger and accretion spin down the black hole.
\end{abstract}

PACS numbers: 04.25.dg, 04.40.Dg, 47.75.+f, 95.30.Sf, 04.30.-w 


\section{Introduction}

\subsection{Motivation}

Black hole-neutron star (BHNS) mergers are expected to be a major source for the upcoming advanced interferometric gravitational-wave detectors (Advanced LIGO, VIRGO, and KAGRA $[1,2,3]$ ), with an expected event rate of the order of 10 BHNS/yr [4] (Note however that this rate is poorly constrained: pessimistic models predict as little as an event every 5 years, while with optimistic assumptions, event rates of up to $300 \mathrm{BHNS} / \mathrm{yr}$ are theoretically possible). If the mergers leave a massive accretion disk around the black hole, they are also a promising setup for short-duration gamma ray bursts (SGRB) [5, 6, 7], which might be observed either directly or as an "orphan" SGRB afterglow. If matter is ejected during the merger (either from the tidal tail or a disk wind), other signals are conceivable, including radio afterglows and/or "kilonovae". (See [8] for a discussion of electromagnetic counterparts.)

It is to be expected that the parameters of BHNS systems will vary widely; the mass and spin of the black hole, in particular, can be substantially different from system to system. Population synthesis studies [9, 10] and mass measurements of stellar-mass black holes [11] both favor black-hole masses of $8-11 M_{\odot}$, but they are currently unable to constrain the spin of the black hole, because this is set mostly by the spin acquired at the hole's formation [9]. This spin at formation will depend on the progenitor of the black hole and the complicated core collapse and accretion dynamics of the black hole's birth; existing simulations can produce a wide range of spins $[12,13]$.

The black-hole spin can strongly affect the outcome of the BHNS merger. Spin decreases the innermost stable circular orbit (ISCO) radius and specific angular momentum for prograde circular orbits, and it increases them for retrograde orbits. It is expected that aligned black-hole spins will increase the mass of the post-merger accretion disk. Numerical simulations confirm this expectation, finding that the disk mass increases dramatically with black-hole spin $[14,15,16]$. Indeed, for BHNS systems with black-hole mass $\gtrsim 10 M_{\odot}$, only those with high dimensionless black-hole spin appear capable of producing significant post-

merger accretion disks $[17,18,19]$. For a low mass system $\left(M_{\mathrm{BH}} \sim 4.5 M_{\odot}\right)$, our previous simulations found that the mass remaining outside of the black hole after merger increased from $5 \%$ of the neutron-star mass for a system with an initially nonrotating black hole to $39 \%$ for a system with a pre-merger black-hole dimensionless spin of $\chi \equiv S / M^{2}=0.9$ [15].

It might appear that this previous study has already effectively covered the range of possible black-hole spins and the consequent post-merger behavior. However, $\chi$ turns out to be a poor measure of how close a black hole is to extremality. For example, a black hole with $\chi=0.9$ has only about half the rotational energy of an extremal black hole of the same mass. More importantly for the accretion phase, the ISCO radius shrinks significantly between $\chi=0.9$ and $\chi=1$ (from $2.3 M_{\mathrm{BH}}$ to $M_{\mathrm{BH}}[20]$ ). Thus, increasing the spin in this range can appreciably change the mass of nuclear matter that is able to avoid prompt 
accretion. It can also increase the efficiency of energy extraction from the accretion disk, $\eta \equiv L / \dot{M} c^{2}$, where $L$ is the luminosity of the disk, $\dot{M}$ is the accretion rate, and $c$ is the speed of light. Taking $\eta$ to be the specific binding energy at the ISCO, as for a thin disk with no ISCO shear, the efficiency for an extremal black hole is higher than that of a $\chi=0.9$ black hole by a factor of 2.8. Finally, we note that for $\chi=0.9$, the ISCO is still outside the ergosphere; the ISCO and, perhaps, significant amounts of disk matter, are only found inside the ergoregion for $\chi>0.94$. It is conceivable that new behavior is possible for these "extreme" cases of accreting $\chi>0.94$ black holes.

Another important drawback of our previous study was that the disk was evolved for only a short time after its formation. A thick accretion disk of nuclear matter with a mass roughly $10 \%$ of the central black hole could show interesting dynamics. If the disk is sufficiently cool, self-gravity could make the disk unstable to spiral density modes or fragmentation. For thin disks, stability is determined by the Toomre parameter $Q_{T}=\kappa c_{s} / \pi G \Sigma[21,22]$, where $c_{s}$ is the sound speed of the disk, $\kappa$ is the epicyclic frequency, $\Sigma$ is surface density, and $G$ is the gravitational constant. Instabilities in thick self-gravitating disks have been investigated numerically in both Newtonian [23, 24, 25, 26, 27] and relativistic [28, 29, 30, 31, 32] physics. The instabilities catalogued in these studies are often very sensitive to the angular momentum profile of the disk, especially the runaway [33, 34] and the Papaloizou-Pringle instabilities [35]. Since the disk is thick and non-Keplerian, its angular momentum profile can only be known by modeling its formation - the BHNS merger, in our case.

Extreme black-hole spin can also profoundly enhance the electromagnetic signal from the post-merger system. The ISCO binding energy is, as we have mentioned, a strongly nonlinear function of $\chi$. Models of neutrino-dominated accretion flows have also shown that a high black-hole spin leads to a higher neutrino luminosity, presumably making more energy available for a gamma ray burst $[36,37,38,39]$. There is also much more rotational kinetic energy in the black hole that can be extracted via the Blandford-Znajek process (with the luminosity $L_{\mathrm{BZ}} \sim \chi^{2}$ ) [40].

\subsection{Summary and overview}

To test the limit of high black-hole spin, high post-merger disk-mass BHNS mergers, we numerically model an extreme case. For the case that we consider, the pre-merger dimensionless black-hole spin is $\chi=0.97$, aligned with the orbital angular momentum. The mass ratio is $3: 1$, corresponding to a low black-hole mass system. The neutron star has a low compaction $M_{\mathrm{NS}} / R_{\mathrm{NS}}=0.144$, corresponding to a neutron-star radius $R_{\mathrm{NS}} \sim 14 \mathrm{~km}$ for a $1.4 M_{\odot}\left(R_{\mathrm{NS}} \sim 12 \mathrm{~km}\right.$ for a $1.2 M_{\odot}$ neutron star $)$. This system is not intended to represent a typical black hole-neutron star binary but is a first step toward exploring the effects of nearly extremal black-hole spin in BHNS mergers. The configuration we consider here illustrates the dynamics possible at one extreme of the astrophysically allowable parameter space, and with this spin and compaction, we can compare to other results to isolate the effects of extreme spin, since the particular combination of mass ratio 3:1 and compaction 
0.144 has been particularly well studied by numerical relativity for lower spins, from $\chi=0$ to $\chi=0.9$. The effects of high black-hole spin could be even more important in BHNS with higher mass ratios or with more compact neutron stars, since systems with high enough mass ratio or low enough compaction would need nearly extremal black-hole spin for the neutron star to tidally disrupt outside the horizon (and thus for the emission of an electromagnetic counterpart to the gravitational-wave signal) [19]. We intend to explore such configurations in future simulations.

We begin our evolution 5.5 orbits $(\approx 21 \mathrm{~ms})$ before merger, when the binary is still in quasicircular orbit. We follow the system until $\approx 27 \mathrm{~ms}$ after merger; this is 13 orbital periods of the maximum density region of the post-merger accretion disk, long enough to let us clearly see the settling of the accretion disk to near equilibrium.

As expected, the extreme spin leads to a very large post-merger accretion disk. Indeed, a full $60 \%$ of the neutron-star matter is able to avoid falling into the black hole during the initial plunge and merger. The vast majority of this matter settles into a massive accretion disk. We note that the early disk mass is significantly larger (by about 50\%) than what was found even for a $\chi=0.9$ system, indicating that the disk mass is very sensitive to the black-hole spin when the spin is nearly extremal.

The matter evolution can be divided into phases, each described in more detail below. First, the neutron star is tidally disrupted, leading to an outgoing tidal tail and an ingoing accretion stream. Second, the accretion stream circles the black hole and collides with itself. This shock rapidly heats the matter and disrupts the accretion stream. Third, the disk settles into axisymmetric equilibrium, starting in the inner regions and proceeding outward. This requires redistribution of the orbital energy and angular momentum to allow fluid elements to settle into circular orbit. In the outer disk, the fluid is nonaxisymmetric and has subequilibrium average angular momentum. The disk accretes into the black hole slowly on a timescale of hundreds of milliseconds.

The rest of this paper is organized as follows. In Sec. 2, we summarize the numerical methods we use to construct and evolve initial data for a black hole and neutron star in a circular inspiral, with the black hole having nearly extremal spin. Then, in Sec. 3, we present and discuss the results of our simulation, including the emitted gravitational waves and the behavior of the black hole's apparent horizon (Sec. 3.1), the behavior of the accretion disk that forms following the neutron star's tidal disruption (Sec. 3.2), and the unbound material ejected by the merger (Sec. 3.3). We briefly conclude in Sec. 4.

\section{Methods}

To construct initial data for a black hole-neutron star binary with nearly extremal blackhole spin, we follow the methods of Ref. [41], which are in part motivated by the methods for constructing binary-black-hole initial data with nearly extremal spins in Ref. [42]. We find that these methods, which had only been tested for spins up to $\chi=0.9$ so far, can

be applied without any modification to generate initial configurations with significantly 


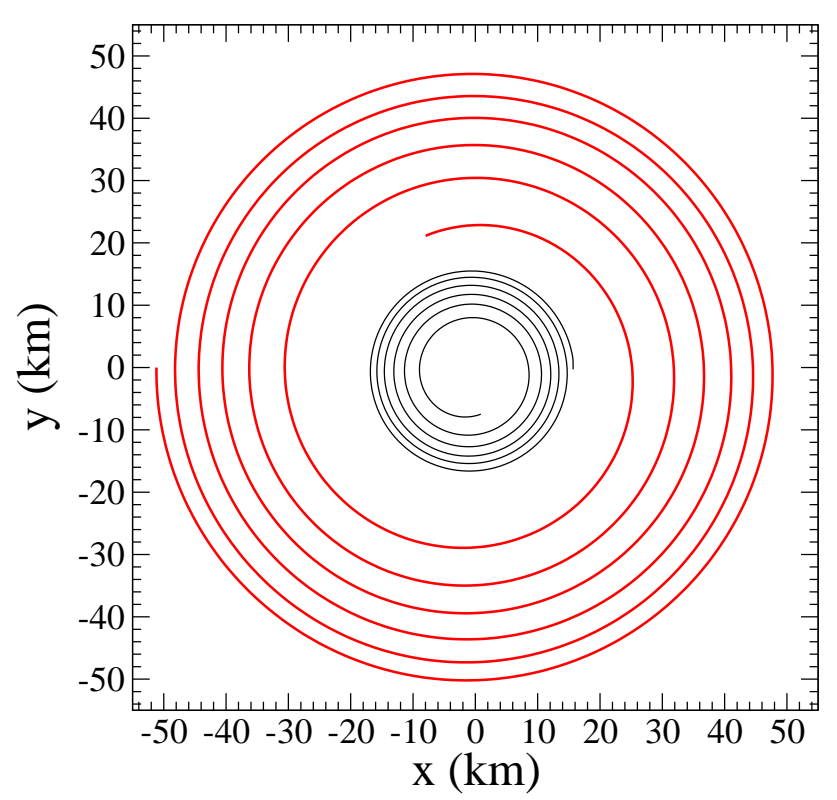

Figure 1. The trajectory of the apparent-horizon center (thin, black curve) and center of mass of the neutron star (thick, red curve) before tidal disruption (i.e., during the first $\approx 20$ ms after the start of the simulation). The orbital eccentricity in this case is $e=0.005 \pm 0.001$.

higher black-hole spins - although obtaining the same accuracy as for lower spins requires the use of a finer numerical grid close to the black hole. Here, we consider a black hole with initial spin $\chi=0.970$. Because the initial conditions are not perfectly in equilibrium, the spin slightly decreases during the initial relaxation, falling to $a / M_{\mathrm{BH}}=0.967$ by time $t / M=100 M_{\odot}=1.5 \mathrm{~ms}$ after the beginning of the simulation (cf. Fig. 2). We use a polytrope

$$
P=\kappa \rho^{\Gamma}+\bar{T} \rho,
$$

where $P$ is pressure, $\rho$ is the rest-mass density, and $\bar{T}$ is a fluid variable related to the physical temperature. As in Ref. [15], we choose $\Gamma=2$ and choose $\kappa$ so that the compaction of the star is $M_{\mathrm{NS}} / R_{\mathrm{NS}}=0.144$. Finally, we reduce the orbital eccentricity of the binary by iteratively solving for its instantaneous angular velocity and inspiral rate [43]. The last iteration, which is used throughout this paper, has eccentricity $e=0.005 \pm 0.001$. The orbital trajectories of the black hole and neutron star before tidal disruption (i.e., during the first $\approx 20 \mathrm{~ms}$ of evolution after the start of the simulation, which corresponds to approximately 5.75 orbits), are shown in Fig. 1.

The coupled evolution of Einstein's equations of general relativity and of the relativistic hydrodynamics equations is performed with the SpEC code [44], using our two-grid method [45]: Einstein's equations are evolved in the generalized harmonic formalism [46], using pseudospectral methods and excision of the black hole interior, while the neutron star fluid is evolved on a separate finite-difference grid covering only the regions in which matter 
is present. The general relativistic equations of hydrodynamics are evolved in conservative form using a second-order finite volume scheme and high-order shock capturing methods: the approximate Riemann problem is solved on cell faces using the Weighted Essentially Non-Oscillatory (WENO5) reconstruction algorithm [47, 48] and Harten, Lax, and van Leer (HLL) fluxes [49]. Both sets of equations are evolved in time using the third-order RungeKutta method with adaptive choice of the time step.

To handle the black hole's high spin during the evolution, we find that we can apply the same techniques that have previously been applied to achieve fully relativistic simulations of merging black hole-black hole $(\mathrm{BHBH})$ binaries with spins of $\chi=0.97[50,51]$. In particular, because we do not impose a boundary condition on the excision surface inside the black hole's apparent horizon, for the numerical evolution to be well-posed we must dynamically adjust the shape and velocity of the excision surface to prevent incoming characteristic fields [52]. During the first $\approx 10 \mathrm{~ms}$ of the evolution, we permitted the excision surface to fall well inside the apparent horizon, provided that there was no danger of incoming characteristic fields, but after noticing that this choice had the undesirable side effect of a noticeable increase in the measured Einstein constraint violation (cf. Fig. 3), for the remainder of the evolution we instead chose to adjust the excision surface so that it tracked the size of the apparent horizon.

We simulated three resolutions through inspiral and tidal disruption, evolving $\approx 27 \mathrm{~ms}$ (low, medium resolution) and $\approx 11 \mathrm{~ms}$ (high resolution) after merger. During the inspiral, these resolutions correspond to approximately $57^{3}, 65^{3}$, and $72^{3}$ spectral gridpoints, respectively, and $100^{3}, 120^{3}$, $140^{3}$ finite-difference gridpoints, respectively. After the neutron star begins to tidally disrupt, we change our domain decomposition, using low, medium, and high resolutions with approximately $98^{3}, 111^{3}$, and $124^{3}$ spectral gridpoints, respectively, and $140^{3}, 160^{3}$, $180^{3}$ finite-difference gridpoints, respectively. From then on, we use spectral adaptive mesh refinement to dynamically adjust the resolution of each spectral subdomain. Note that for all evolutions, since the black-hole spin is aligned with the orbital angular momentum, when solving the relativistic hydrodynamic equations we only evolve the region above the orbital plane, imposing a symmetry condition on the orbital plane and reducing the number of gridpoints in the direction normal to the orbital plane by a factor of 2 .

We note that we do not find it necessary or advantageous to change to a damped harmonic gauge condition, as was done in the high-spin BHBH simulations in Refs. [50, 51]. Instead, we use the same gauge conditions used in previous two-grid BHNS evolutions at the same mass ratio [15].

These simulations are computationally expensive. The low, medium, and high resolutions cost approximately $9.8 \times 10^{4}, 1.4 \times 10^{5}$, and $1.5 \times 10^{5} \mathrm{CPU}$ hours, corresponding to approximately 70, 105, and 116 days of wallclock time. (Note that because we did not continue the high resolution simulation for as long after disruption, its expense is only slightly higher than that of the medium resolution.) 


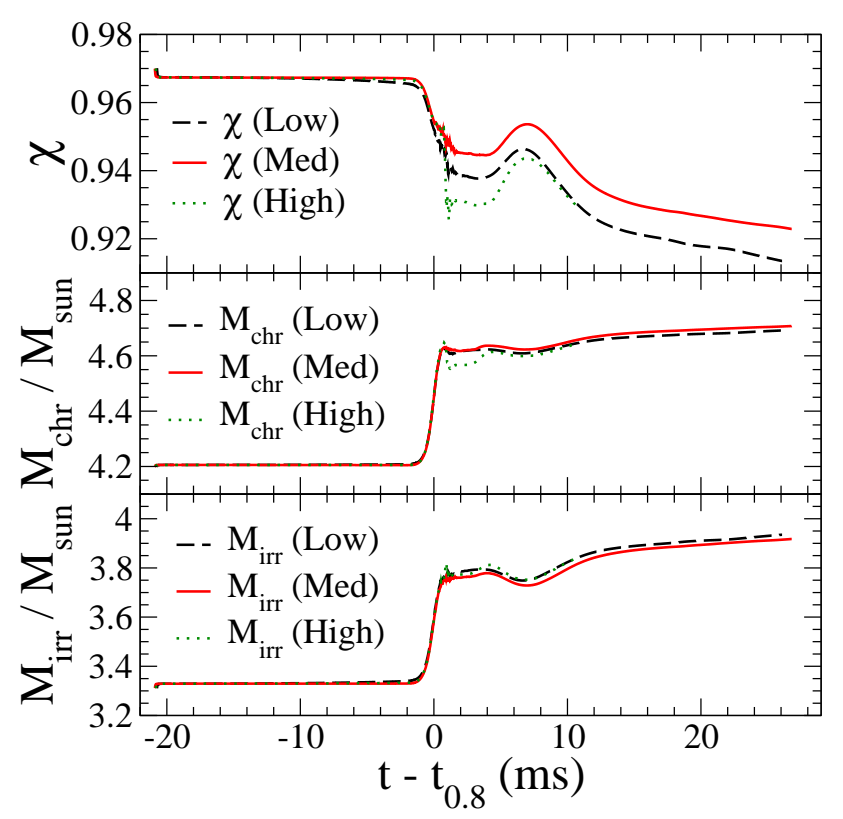

Figure 2. The evolution of the black-hole spin $\chi:=S / M_{\mathrm{chr}}^{2}$ (top panel), Christodoulou mass $M_{\text {chr }}$ (middle panel), and irreducible mass $M_{\text {irr }}$ (bottom panel) as a function of time (with times $t$ shown relative to time $t_{0.8}$, the time when the baryonic mass outside the hole has fallen to $80 \%$ of its initial value) for a black hole-neutron star merger with an initial black hole spin of magnitude $\chi=0.97$.

\section{Results}

In this section, we present our main numerical results. First, in Sec. 3.1, we show results for the spacetime's evolution. Then, we discuss the properties of the resulting accretion disk (Sec. 3.2) and ejecta (Sec. 3.3) after the black hole tidally disrupts the neutron star.

\subsection{Spacetime}

To characterize the behavior of the curved spacetime, we begin by examining the mass and spin of the black hole as measured on the apparent horizon. Figure 2 shows, for each resolution, the black-hole dimensionless spin $\chi:=S / M_{\mathrm{ch}}^{2}$ (top panel), Christodoulou mass $M_{\mathrm{ch}}:=\sqrt{M_{\mathrm{irr}}^{2}+S^{2} /\left(4 M_{\mathrm{irr}}^{2}\right)}$ (middle panel), and irreducible mass $M_{\mathrm{irr}}$ (lower panel) as functions of time. Following the initial relaxation, the masses increase slightly (from $M_{\text {irr }}=3.31$ and $M_{\mathrm{ch}}=4.20$ to $M_{\mathrm{irr}}=3.33$ and $\left.M_{\mathrm{ch}}=4.21\right)$ while the dimensionless spin decreases slightly (from $\chi=0.970$ to $\chi=0.967$ ). The masses and spins then remain constant in time to within our numerical accuracy until approximately $20 \mathrm{~ms}$ after the start of the simulation, when the neutron star begins to disrupt. During the next $\approx 1 \mathrm{~ms}$, the masses sharply increase, while the dimensionless spin sharply decreases. The hole's masses then continue to slowly increase as its dimensionless spin slowly decreases.

While all three resolutions exhibit the same qualitative behavior, we were unable to 


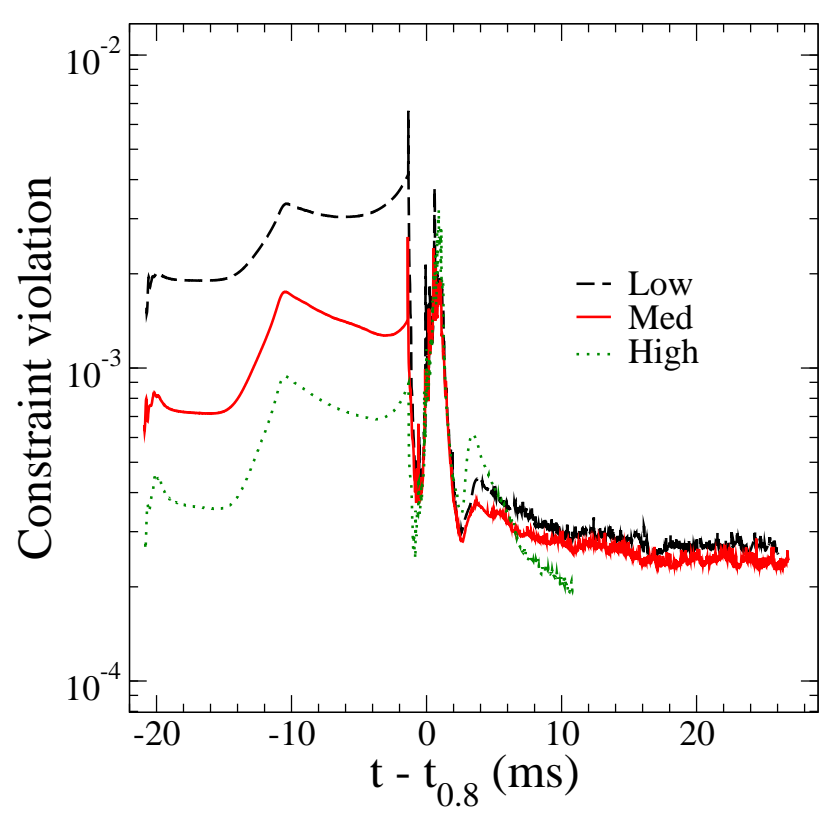

Figure 3. The normalized Einstein constraint violation (as defined in Eq. (71) of Ref. [46]) as a function of time (with times $t$ shown relative to time $t_{0.8}$, the time when the baryonic mass outside the hole has fallen to $80 \%$ of its initial value) for low (dashed, black), medium (solid, red), and high (dotted, green) resolutions. In each simulation, the initial black-hole spin is aligned with the orbital angular momentum and has a magnitude of $\chi=0.97$. The constraints rise around time $t-t_{0.8}=-14 \mathrm{~ms}$ because the apparent horizon is moving farther from the excision surface. After modifying the simulation's algorithm so that the excision surface and apparent horizon would nearly coincide (at approximately $t-t_{0.8}=-10 \mathrm{~ms}$ ), the constraint violation drops until the neutron star begins to disrupt.

demonstrate convergence of the black hole's mass and spin through the end of the simulation. Shortly after disruption begins, in the high-resolution simulation the black hole experiences an anomalously large decrease in the dimensionless spin; through experimentation, we found this decrease to be sensitive to the details of dynamic regridding (i.e., the algorithm used to resize the finite-difference grid as the region containing matter expands - cf. Sec. II B 1 of Ref. [15]). This could be a consequence of the extremely large amount of mass remaining in the disk. Apparently, there are certain special distributions of matter on our grid that, upon a regrid, result in unexpectedly large interpolation error near the excision surface and a corresponding small but abrupt change in the evolution. Since our primary concern in this paper is the behavior of the disk and ejecta, and since this $O(\%)$ effect does not qualitatively change our resultsł, we have chosen to leave our efforts to resolve the black-hole spin after

$\ddagger$ We are confident that this anomaly does not qualitatively affect our results or our conclusions, for the following reasons: i) Cf. the top panel of Fig. 10, which shows the result for all three $\chi=0.97$ resolutions: all three resolutions show the same qualitative behavior; ii) The $O(\%)$ anomalous behavior seen in the highresolution black-hole quantities around time $t-t_{0.8} \approx 1 \mathrm{~ms}$ coincides with a period of the evolution when the finite-difference grid rapidly, repeatedly regrids. The effect of these regrids can also be seen in small discontinuous changes in disk properties such as the baryon rest mass. Disabling regridding did eliminate 
disruption (perhaps by improving our regridding method) for future work.

Figure 3 further demonstrates our difficulty in obtaining convergence after tidal disruption. Before disruption (i.e. during the first $20 \mathrm{~ms}$ of the simulation), the normalized constraint violation appears to be convergent, as expected. The constraints do rise around $t-t_{0.8} \approx-14 \mathrm{~ms}$ as the excision surface falls far inside the apparent horizon, but this trend reverses after a modification of our method to keep the excision surface close to the apparent horizon§. After interpolating to our higher-resolution domain (around $t-t_{0.8} \approx-2 \mathrm{~ms}$ ), the constraints sharply fall at first, but then sharply spike during the merger. The subsequent bump in the constraints corresponds to the small bump in the dimensionless spin and the small drop in irreducible mass seen around $t-t_{0.8} \approx 7 \mathrm{~ms}$ in Fig. 2.

We conclude this section by examining the dominant mode of the gravitational waveform (Fig. 4). Because we plot $\Psi^{4}=\left(d^{2} / d t^{2}\right)\left(h_{+}-i h_{\times}\right)$instead of the wave amplitude $h$, the spurious "junk" gravitational radiation emitted during the initial relaxation is clearly visible. Each resolution shows a small, secondary burst of gravitational waves after the primary wave has terminated. The phase of this secondary burst cannot be resolved in the simulation: as is typical for numerical simulations of BHNS or binary neutron star mergers, the phase error in the post-merger waveform is on the order of a few radians. At all resolutions, the burst carries an energy $E_{\text {burst }} \sim 10^{-4} M_{\odot} c^{2}$ in a time $t_{\text {burst }} \sim 0.75 \mathrm{~ms}$ and at frequencies $f_{\text {burst }} \sim 2-3 \mathrm{kHz}$. There are no easy ways to associate this burst in the gravitational wave signal extracted at large radius with a specific feature of the forming accretion disk. Additionally, sharp features due to shocks will only converge at first order with numerical resolution, and their effects are likely to be poorly resolved. Nonetheless, an estimate from the quadrupole formula shows that these numbers are consistent with emission from an overdense region with excess mass $M_{\text {emitter }} \sim 0.01-0.03 M_{\odot}$, orbiting in the inner region of the disk $(R \sim 30 \mathrm{~km})$. For the massive disk observed here, the presence of such an asymmetry immediately after merger is quite natural. We thus expect these features to be qualitatively correct, even though the detailed properties of the waveform are not numerically resolved.

\subsection{Accretion disk}

After tidal disruption, the nuclear matter forms an outgoing tidal tail and an ingoing accretion stream. At a time $t=t_{0.8}+10 \mathrm{~ms}(10 \mathrm{~ms}$ after the time when $80 \%$ of the

the anomalous behavior in the high-resolution run. Unfortunately, long evolutions cannot be done without regriddng. The frequent regridding behavior that seems to cause the problem is not seen in the low and medium resolution runs. iii) The low and medium resolutions, which don't display the frequent regridding problem, show good quantitative agreement with each other.

$\S$ Because of the high cost of our simulations (cf. Sec. 2), we chose to adjust our algorithm during the simulations - at a time early enough that the constraint violation is still small - rather than to repeat the simulations from the beginning. We observe no significant effect corresponding to this adjustment in our results; therefore, we are confident that this adjustment does not affect our conclusions. However, had we continued to allow the excision surface to remain far inside the apparent horizon, constraint growth would eventually (before the time of merger) have caused the simulations to fail. 


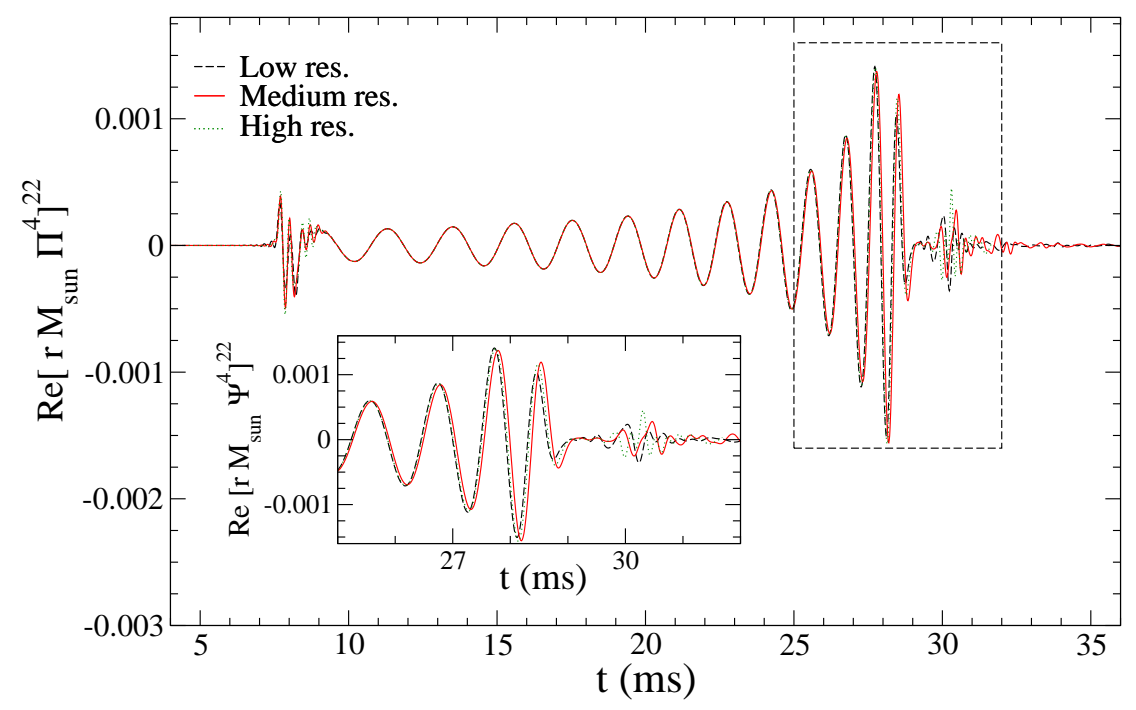

Figure 4. The real part of the dominant $\ell=m=2$ mode of the gravitational waveform $r M_{\text {sun }} \Psi^{4}$, where $r$ is the radius of the sphere on which the gravitational waveform is extracted (here the outermost sphere, with a radius of $r=1540 M_{\odot}$ ), $M_{\text {sun }}=1 M_{\odot}$, and $\Psi^{4}$ is the Newman-Penrose scalar corresponding to outgoing gravitational waves. The inset zooms in on the part of the waveform enclosed by the dashed box.

initial baryonic mass remains outside the hole), $\approx 23 \%$ of the initial baryonic mass has moved outward and left the finite-difference grid, and $\approx 31 \%$ of the initial baryonic mass remains on the finite-difference grid (Fig. 5). Of the mass that has left, $\approx 17 \%$ appears to be unbound and quickly leaves the grid (see Sec. 3.3), while another $\approx 6 \%$ is weakly bound but extends far enough from the black hole to leave the finite-difference grid. This material would have fallen back onto the disk at later times $\left(\sim 10^{2} \mathrm{~ms}\right)$ and its loss is one reason we limit our simulations to the early to middle $(\leq 30 \mathrm{~ms})$ post-merger evolution.

During this tidal disruption phase, the matter remains cold and degenerate. The infalling stream of matter circles around the black hole and collides with itself, producing an intense shock that travels through and disrupts the incipient accretion flow. Nearly all of the entropy generation in the evolution occurs during this crucial millisecond, which could be labeled the shock phase of the evolution. Shock heating renders the matter completely nondegenerate, and hot matter flows to the outer disk region $(r>100 \mathrm{~km})$ over the next $\sim 10 \mathrm{~ms}$. By the end of this time, a clear hierarchy of velocities establishes itself through the disk out to $200 \mathrm{~km}$. (See Fig. 6.) Inside this region, the azimuthal velocity dominates and is roughly twice the sound speed throughout $\left(\Omega r \approx 2 c_{s}\right)$. The orbital period sets the dynamical timescale; we may define a "settling radius" $r_{\text {settle }}(t)$ as the radius at which gas has had time to complete exactly one orbital period since merger. Since orbits will not fully circularize after one period, this radius provides only a rough sense of which parts of the disk have had time to settle. By the end of our evolution (30 ms postmerger), $r_{\text {settle }}=190$ $\mathrm{km}$. Outside $r_{\text {settle }}$, the disk does not have time to reach equilibrium. The radial velocity 


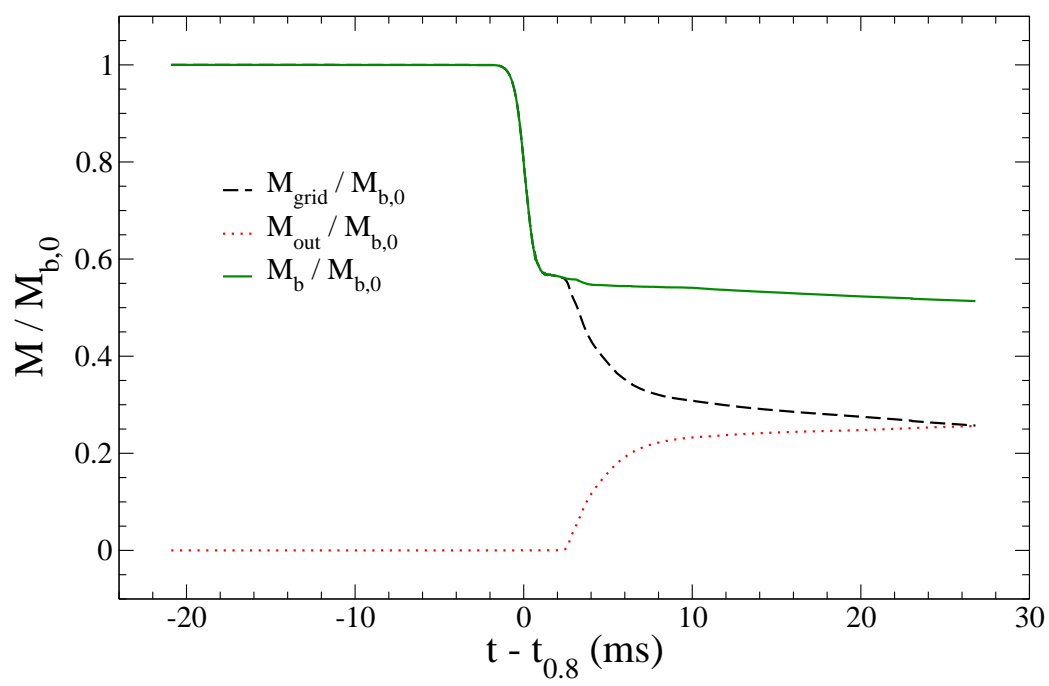

Figure 5. The evolution of the baryonic mass $M_{\mathrm{b}}$ as a function of time $t$ for the medium resolution black hole-neutron star merger with an initial aligned black-hole spin of magnitude $\chi=0.97$. The solid, green line is the total baryonic mass $M_{\mathrm{b}}$ outside the black hole, the dashed, black line is the total rest mass $M_{\text {grid }}$ remaining on the finitedifference computational grid, and their difference $M_{\text {out }}$ is the dotted, red line. All masses are normalized by the baryonic mass $M_{b, 0}$ at the start of the simulation. Times are shown relative to time $t_{0.8}$, the time when the baryonic mass outside the hole has fallen to $80 \%$ of its initial value.

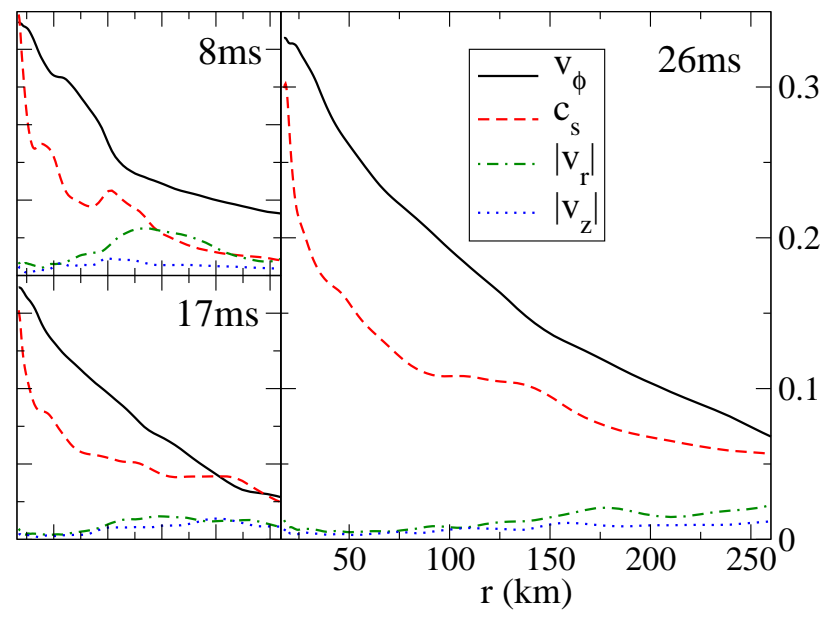

Figure 6. A break-up of the components of the fluid velocity, shown at three times after merger, as a fraction of the speed of light. Also shown is the sound speed $c_{s}$. The extents on the horizontal and vertical axes are the same in all three panels. The radial velocity itself is negative (infall). 


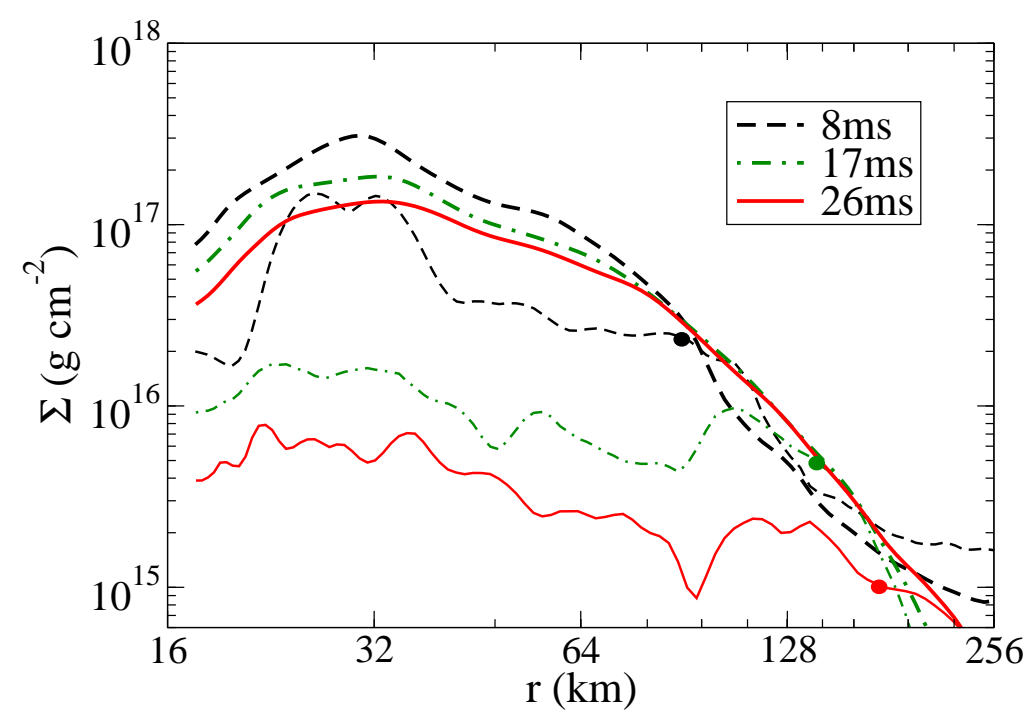

Figure 7. The density profile of the accretion disk, shown at times $t=8,17$, and $26 \mathrm{~ms}$ after merger. For each time, two lines are shown. The top line is the vertically integrated surface density $\Sigma$ at each radius, averaged over all angles. The bottom line is the rms deviation from the average due to nonaxisymmetry. A dot on the bottom line indicates the location of $r_{\text {settle }}$ at this time. In this and all other radial plots, the radius shown is constructed from the proper circumference at the equator.

is a nonnegligible fraction of the azimuthal velocity in the outer disk (see Fig. 6), but the radius at which this infall becomes supersonic recedes with $r_{\text {settle. }}$.

In Figure 7, we show the surface density $\Sigma$ at three times during the settling phase. The density peaks at a circumferential radius of around $30 \mathrm{~km}$; the location of the peak remains fixed, while the overall density slowly decreases everywhere as the mass is depleted by accretion. Also shown in this plot is the RMS deviation in $\Sigma$ at each radius due to deviations from axisymmetry. We see that the inner disk is quite axisymmetric, but that deviations from axisymmetry reach order unity at around $r_{\text {settle }}(t)$. A Fourier analysis of $\Sigma$ at late times shows that the deviation from axisymmetry primarily subsists in trailing spiral features, although localized twists in which a mode is leading over a small radial range are occasionally seen in some modes. The dominant mode in the outer disk is $m=1$. Only in the inner region can these modes be regarded as linear perturbations of an equilibrium system; here they provide a natural way to understand the extraction of angular momentum from the inner disk which must occur if the low-angular-momentum outer disk (see below) is to settle. In addition to these smooth modes, equilibrium in $r_{\text {settle }} / 2<r<r_{\text {settle }}$ is sometimes disturbed by weak, localized sharp features, presumably weak shocks where gas on eccentric orbits hits the more settled disk. These features are most clearly seen in the pressure force (see below).

In Figure 8, we plot the specific orbital energy $\left(E=u_{t}\right)$ and angular momentum $\left(L=-u_{\phi} / u_{t}\right)$ on the equator as a function of radius. For comparison, we plot the expected 
curves for circular orbit geodesics in this spacetime metric. The geodesic $E$ and $L$ are the expected orbital parameters for a stationary disk taking into account the disk's self-gravity (which is included in the numerical metric) but not pressure forces. Except in the very inner disk, we see significant deviations from geodesic orbits, as would be expected for a thick disk. Figure 8 also includes $E$ and $L$ for equilibrium circular orbits, i.e. stationary circular orbits taking into account the pressure force for the given fluid profile. These agree with the actual $E$ and $L$ up to about $100 \mathrm{~km}$. Beyond this, the energy curves continue to agree (accounting for deviations due to localized shocks), but the angular momentum profiles flatten more rapidly than the equilibrium curve (although the latter seems to become somewhat noisy) and become sub-equilibrium. The gas at these radii was ejected (from the tidal tail or the post-merger shock) into eccentric orbits, as seen from the $L$ deficit and the nonzero radial velocity (cf. Fig. 6). Equivalently, the nonaxisymmetric modes carry net negative angular momentum. Pressure support becomes especially important in the outer disk, as can be seen from the deviation between geodesic and equilibrium curves. We also have observed that the disk thickness, height $H$ divided by radius $r$, increases with radius from about $H / r \approx 0.2$ in the inner disk to about $H / r \approx 0.35$ in the outer disk.

There are no clear signs of instability or turbulence in the disk, a fact that itself should be explained. The lack of obvious global corotation instability of the kind found in some other massive disk simulations is a natural consequence of the angular momentum profile. Instability is only expected for $L \propto r^{n}, n<2-\sqrt{3}$ [35] with growth rate decreasing rapidly as $n$ increases above zero, and our disk has $n \approx 0.3$ in the equilibrated region. This region is also stable against the effects of self-gravity, with Toomre parameter no lower than $Q_{T} \approx 400$. This stability is a consequence both of the shear and the thermal pressure. Radiative cooling may later remove some of the disk's heat, but even removing all of it would leave $Q_{T} \sim 10$. Shear and thermal energy also conspire to protect the disk from convective instability. In Newtonian physics, the Solberg-Hoiland stability criteria are

$$
\begin{aligned}
& r^{-3} \nabla_{r} L^{2}-\left(C_{p} \rho\right)^{-1} \nabla P \cdot \nabla S>0 \\
& \nabla_{z} P\left(\nabla_{r} L^{2} \nabla_{z} S-\nabla_{z} L^{2} \nabla_{r} S\right)<0,
\end{aligned}
$$

where $\rho$ is the density, $S$ the entropy, $P$ the pressure, and $C_{P}$ the heat capacity. The relativistic version of these equations is slightly more complicated but basically similar [53]. Figure 9 shows the average specific entropy as a function of radius during the settling phase. The entropy has a minimum close to the density maximum, while the gas on the edges is hotter. Thus $\nabla P \cdot \nabla S<0$ except near a small ring near $r=40 \mathrm{~km}$, and this feature is stabilized by the strong shear. In fact, the epicyclic frequency is much greater than the Brunt-Väisälä frequency $N$ out to $r \approx 80 \mathrm{~km}$. Beyond this, the $S$ gradient steepens, the $L$ gradient flattens, and buoyancy becomes an important restoring force. Vertical convection is also suppressed by a strong positive $\nabla_{z} S$. The disk will be unstable to the magnetorotational instability, since $d \Omega^{2} / d \ln r+N^{2}<0$ throughout, but this does not appear in our simulations, which do not include magnetic fields.

The disk evolves slowly due to the settling of the outer disk and accretion of gas in the 


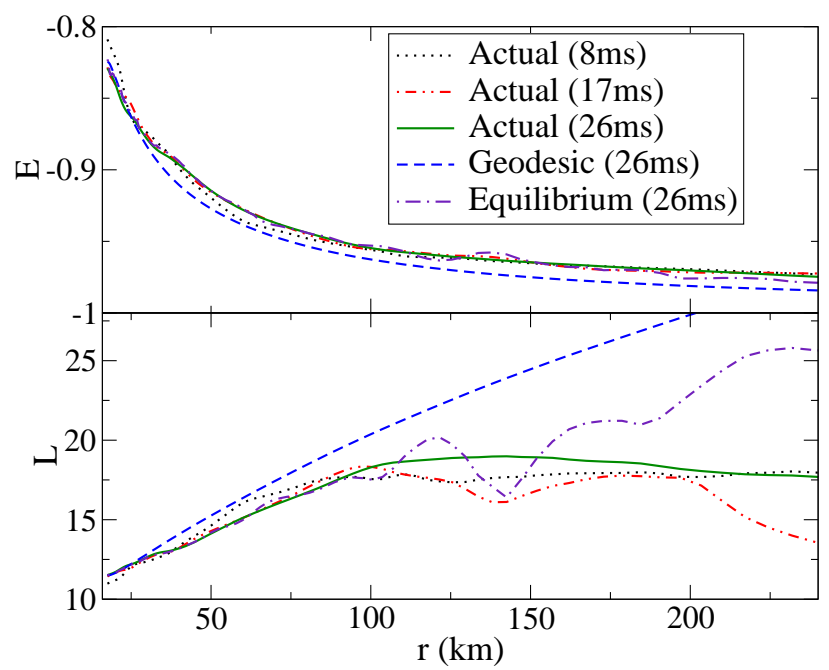

Figure 8. The specific orbital energy $E$ and specific angular momentum $L$ at three times after merger. For the final time, we include two other curves for comparison. First, we include $E$ and $L$ for circular orbit geodesics in the given metric (which will include the disk's self gravity). Second, we show $E$ and $L$ for equilibrium circular orbits given the current metric and the pressure forces from the current pressure and density profiles. For the equilibrium energy, we take the median in angle rather than the mean. The mean would reveal spikes at $140 \mathrm{~km}$ and $190 \mathrm{~km}$ due to local shock features. The geodesic curves for the earlier times are nearly identical to those at late times, while the equilibrium curves differ only at large radii.

inner disk (the two perhaps connected by transfer of angular momentum). The late-time accretion rate is $\dot{M} \approx 2 M_{\odot} \mathrm{s}^{-1}$, giving a lifetime of $\tau \equiv M / \dot{M} \sim 200 \mathrm{~ms}$. Before this time, we expect magnetorotational effects to become important. The most unstable modes will grow on the dynamical timescale (ms), and then magnetic turbulence will transport angular momentum. Assuming this acts like an $\alpha$ viscosity with $\alpha=0.01-0.1$, the effective viscous timescale will be of order $r^{2} /\left(\alpha c_{s} H\right) \sim 10-100 \mathrm{~ms}$ for radius $r$, height $H$, and sound speed $c_{s}$ characteristic of the central torus. Accretion increases the spin and mass of the black hole, but since the accreted matter has low angular momentum $(L \approx 10$, see Fig. 8$)$, the total effect on the dimensionless spin $\chi=S / M^{2}$ will be small even for accretion of most of the disk mass. (At late times in our evolution, $\chi$ is slowly decreasing.)

Our simulation does neglect several effects that could influence the accretion disk's later evolution. A magnetic field could produce a jet, powered either by the accretion flow or the high black hole spin. It would certainly render our disk magnetorotationally unstable. Although we find that MRI turbulence is not needed for strong early-time accretion, it will still alter the disk evolution in important ways. Second, neutrino radiation will cool the gas and make it less neutron-rich. Assuming a simple ideal gas plus radiation pressure equation of state, we infer that the temperature in the disk is of order $10 \mathrm{MeV}$. (In this model, the disk is gas pressure-dominated everywhere except the inner edge.) Using a simple diffusion 


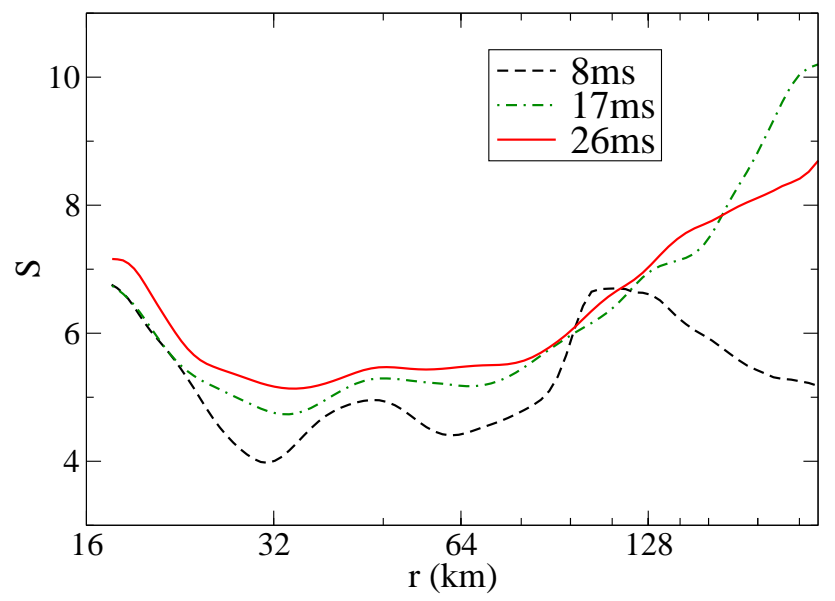

Figure 9. The entropy profile of the accretion disk, shown at times $t=8,17$, and $26 \mathrm{~ms}$ after merger. The entropy for our $\Gamma$-law equation of state is $S=\log \left(\frac{P(\rho, T)}{P(\rho, 0)}\right)$. For each radius, a density-weighted average is carried out over the $\phi$ and $z$ coordinates.

approximation, one can estimate a luminosity $L_{\nu} \sim 10^{52} \mathrm{erg} \mathrm{s}^{-1}$, which could deplete the disk's thermal energy on a timescale of $\sim 10^{2} \mathrm{~ms}$. More likely, the cooling will balance the dissipative heating introduced by magnetic turbulence. Neutrino radiation could itself induce unstable entropy or composition gradients and drive convection. Finally, our grid limitations have forced us to allow gravitationally bounded, outward-moving material to leave the finite-difference grid. This matter should eventually fall back onto the disk, perturbing and perhaps shocking it. Tracking the fallback matter will be a computational challenge for future simulations that wish to evolve disks like this one further in time than was done here.

We should note that the amount of matter remaining outside of the black hole after merger is not only larger than in any previous studies of BHNS mergers: it is also significantly above the predictions obtained by extrapolating results for lower spin black holes in the high spin regime. The remnant mass for lower spin systems is generally well approximated by [19]

$$
\frac{M_{\mathrm{rem}}}{M_{\mathrm{NS}}}=0.288\left(\frac{3 M_{\mathrm{BH}}}{M_{\mathrm{NS}}}\right)^{1 / 3}\left(1-2 \frac{M_{\mathrm{NS}}}{R_{\mathrm{NS}}}\right)-0.148 \frac{R_{\mathrm{ISCO}}}{R_{\mathrm{NS}}},
$$

with $R_{\mathrm{ISCO}}$ the radius of the innermost stable circular orbit around the black hole. Figure 10 shows the predicted remnant mass for BHNS mergers at $q=3, M_{\mathrm{NS}} / R_{\mathrm{NS}}=0.144$, together with numerical results at $\chi=(0,0.5,0.75,0.9,0.97)[14,18]$. We see that the analytical predictions perform well for low-spin black holes $(\chi<0.9)$, while strong deviations are visible for the higher spin configurations. This is not surprising: the analytical results are only supposed to be valid for relatively low mass remnants $\left(M_{\text {rem }}<0.2 M_{\odot}\right)$. But it does emphasize the need for further studies of BHNS mergers at high black-hole spin in order to properly model the characteristics of the post-merger remnant in that regime - especially considering that the most energetic SGRBs observed might require the presence of such high-mass accretion disks [54]. 

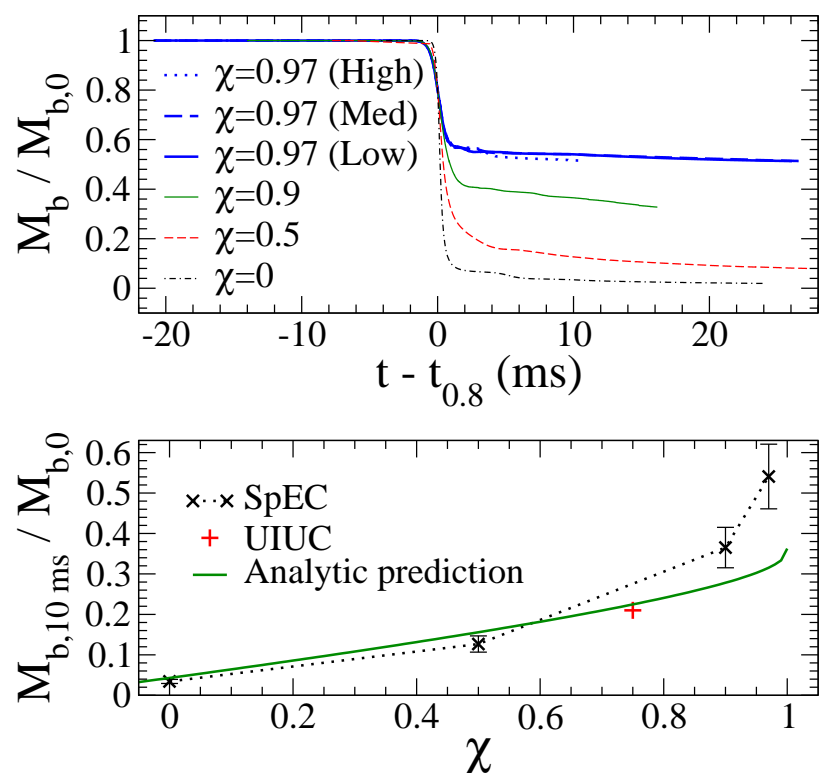

Figure 10. The evolution of the baryonic mass for black hole-neutron star mergers with different initial black-hole spins of magnitude $\chi$ aligned with the orbital angular momentum. The mass for $\chi=0.97$ is shown for the three different resolutions (low, medium, high) simulated. The masses from mergers with $\chi=0, \chi=0.5$, and $\chi=0.9$ are taken from Fig. 5 of Ref. [15]. Top panel: The baryonic masses are shown as a function of time $t-t_{0.8}$, where $t_{0.8}$ is the time at which the baryon mass outside the hole has fallen to $80 \%$ of its initial value. Bottom panel: The baryonic mass at time $t=t_{0.8}+10 \mathrm{~ms}$ (black crosses) as a function of the initial black-hole spin. The medium resolution of the $\chi=0.97$ simulation is shown. Relative errors of $15 \%$ are used for all simulations, an estimate obtained by extrapolating our results to infinite resolution while assuming second order convergence of the code. For comparison, also shown are i) the final disk mass obtained by the UIUC group in Fig. 13 of Ref. [14] for case "B" and ii) the theoretical prediction derived in Ref. [19].

\subsection{Ejecta}

In addition to the formation of an accretion disk, the tidal disruption of the neutron star can lead to the ejection of a significant amount of unbound material. This material is initially neutron rich, and its radioactive decay can lead to detectable optical afterglows ("kilonovae" $[55,8]$ ), as well as contribute to the formation of heavy elements (r-process nucleosynthesis). The deceleration of the ejecta in the interstellar medium can also cause emission in the radio band [8]. For binary neutron stars [56] and low-spin BHNS [57] binaries, general relativistic simulations show that only a small fraction of the neutron star material $(<1 \%)$ is unbound, with kinetic energies of $10^{49} \mathrm{ergs}-10^{51} \mathrm{ergs} \mathrm{[56].}$

Measurements of the unbound mass are fairly inaccurate in our code, especially for very energetic and massive ejecta. This is due to the fact that the fluid equations are only evolved up to $20 M_{\mathrm{BH}}-30 M_{\mathrm{BH}}$ from the center of mass of the system, while measurements of the properties of the ejecta are best done far away from the black hole and in low-density tidal tails (the condition $u_{t}<-1$, used to determine whether material is unbound, is only valid 
for a stationary metric and pressureless fluid). Additionally, the accuracy of the evolution far away from the black hole is lower than in the forming disk, where most grid points are concentrated. The development of techniques allowing us to efficiently evolve the fluid equations at larger distances without losing accuracy close to the black hole (e.g. adaptive mesh refinement) will be required to avoid these issues. In a recent paper [58], we showed that the main source of error when measuring the mass of the ejecta is generally the large grid spacing in the far zone. The same holds in this simulation: we find

$$
M_{\mathrm{ej}}=0.26 M_{\odot} \pm 0.16 M_{\odot}
$$

assuming 2nd order convergence between the medium and high resolution. This confirms that BHNS mergers with rapidly rotating black holes are significantly more favorable to the ejection of neutron-rich material. In [58], we found that for higher mass ratio systems $(q=7)$ and lower spins $\chi=0.9$, a few percent of a solar mass is likely to be unbound, already a noticeable improvement compared to low-spin systems. The ejecta in the case studied here, which is of course an extremely favorable configuration, is an order of magnitude larger thus showing that for rapidly spinning systems, it is conceivable that a large fraction of the mass remaining outside of the black hole at late time is unbound.

Measurements of the kinetic energy of the ejecta are even less reliable than its mass. Indeed, the kinetic energy is dominated by the most relativistic parts of the ejecta, which are also the most poorly resolved. From measurements of $u_{t}$ as the outward-moving material is leaving the finite-difference grid, we estimate that the ejected material has a median velocity $v \sim 0.5 c$, and kinetic energy $E_{\text {ej }} \sim 10^{52} \mathrm{ergs}-10^{53} \mathrm{ergs}$. These numbers should, however,

be considered only as order of magnitude estimates. Nonetheless, this is an extremely large amount of energy, which would cause the emission of a detectable radio signal for a large fraction of the mergers within the range of Advanced LIGO as the ejecta slows down in the interstellar medium.

\section{Conclusion}

We have simulated the merger of a black hole-neutron star system with a premerger blackhole spin parameter of $\chi=0.97$, the highest spin yet attempted for modeling such systems. Given the strongly nonlinear dependence of many aspects of the merger on $\chi$ (black-hole rotation energy, ISCO location, accretion efficiency), this simulation carries the numerical exploration of BHNS mergers into a distinctly new regime not sampled even by our previous simulations with $\chi=0.9$. These nonlinearities manifest themselves clearly in our results.

Upon disruption of the neutron star, less than half of the nuclear matter is promptly accreted into the black hole. Almost 20\% appears to be ejected from the system in an unbounded tidal tail outflow, and the rest settles into a massive accretion disk. Both the ejecta and disk masses are higher than any previous fully relativistic BHNS simulation. This in itself is unsurprising; both theoretical considerations and previous simulations would lead us to expect that in higher- $\chi$ systems more matter should evade prompt accretion. 
What is notable is that the disk mass exceeds even the expectations for this spin based on extrapolating the trends of lower-spin systems. If such systems occur in nature, they must be particularly spectacular events even by the standard of BHNS mergers.

We have closely followed the behavior of the horizon and of the accretion disk. Both merger and accretion have the net effect of decreasing the dimensionless spin $\chi$ of the black hole. While the disk is thick and self-gravitating, it appears to be quite stable, settling to an axisymmetric quasistationary state and evolving only slowly under the influences of outer-disk settling and accretion-induced mass decrease. Its subsequent evolution will be driven in part by physical processes not included in these simulations.

That existing numerical relativity techniques can successfully treat such an extreme system essentially unaltered is encouraging. Further studies of the extreme-spin regime of BHNS parameter space can now be attempted; particularly interesting will be the exploration of systems with higher black-hole mass. These are expected to be more common astrophysically, and they may also be more intriguing from a relativist's point of view, since the disruption event happens nearer to the horizon, as measured by the disruption separation divided by $M_{\mathrm{BH}}$. The investigation of the accretion system studied in this paper should also be completed by incorporating the remaining crucial physics, particularly the magnetic turbulence.

\section{Acknowledgments}

We are pleased to thank Christian Ott, Robert Owen, and Saul Teukolsky for helpful discussions. This work was supported in part by grants from the Sherman Fairchild Foundation to Cornell and Caltech, by NSF Grants No. PHY-0969111 and No. PHY1005426 at Cornell; by NSF Grants No. PHY-1068881 and PHY-1005655 at Caltech; by NASA Grant No. NNX09AF96G at Cornell; and NASA Grant No. NNX11AC37G and NSF Grant PHY-1068243 to WSU. The numerical computations presented in this paper were performed primarily on the Caltech compute cluster ZWICKY, which was funded by the Sherman Fairchild Foundation and the NSF MRI-R ${ }^{2}$ grant No. PHY-0960291 to Caltech.

\section{References}

[1] The LIGO scientific collaboration. The science of LSC research. http://www.ligo.org/science/overview.php.

[2] The Virgo Collaboration. Advanced Virgo Baseline Design, 2009. [VIR-0027A-09].

[3] K. Somiya and the KAGRA Collaboration. Detector configuration of KAGRA-the japanese cryogenic gravitational-wave detector. Class. Quantum Grav., 29(12):124007, 2012.

[4] J. Abadie, B. P. Abbott, R. Abbott, M. Abernathy, T. Accadia, F. Acernese, C. Adams, R. Adhikari, P. Ajith, B. Allen, and et al. TOPICAL REVIEW: Predictions for the rates of compact binary coalescences observable by ground-based gravitational-wave detectors. Classical and Quantum Gravity, 27(17):173001, September 2010.

[5] B. Paczynski. Cosmological gamma-ray bursts. Acta Astronomica, 41:257-267, 1991. 
[6] R. Narayan, B. Paczynski, and T. Piran. Gamma-ray bursts as the death throes of massive binary stars. Astrophys. J. Lett., 395:L83-L86, August 1992.

[7] H.-Th. Janka, T. Eberl, M. Ruffert, and C. L. Fryer. Black hole-neutron star mergers as central engines of gamma-ray bursts. Astrophys. J., 527:L39, 1999.

[8] B. D. Metzger and E. Berger. What is the Most Promising Electromagnetic Counterpart of a Neutron Star Binary Merger? Astrophys. J., 746:48, February 2012.

[9] K. Belczynski, R. E. Taam, E. Rantsiou, and M. van der Sluys. Black Hole Spin Evolution: Implications for Short-Hard Gamma-Ray Bursts and Gravitational Wave Detection. Astroph.J., 682:474-486, July 2008.

[10] K. Belczynski, M. Dominik, T. Bulik, R. O'Shaughnessy, C. Fryer, and D. E. Holz. The Effect of Metallicity on the Detection Prospects for Gravitational Waves. Astrophys. J. Lett., 715:L138-L141, 2010 .

[11] F. Özel, D. Psaltis, R. Narayan, and J. E. McClintock. The Black Hole Mass Distribution in the Galaxy. Atrophys.J., 725:1918-1927, December 2010.

[12] Y. Sekiguchi and M. Shibata. Formation of Black Hole and Accretion Disk in a Massive High-entropy Stellar Core Collapse. Astrophys. J., 737:6, August 2011.

[13] C. D. Ott, C. Reisswig, E. Schnetter, E. O'Connor, U. Sperhake, F. Löffler, P. Diener, E. Abdikamalov, I. Hawke, and A. Burrows. Dynamics and Gravitational Wave Signature of Collapsar Formation. Physical Review Letters, 106(16):161103, April 2011.

[14] Zachariah B. Etienne, Yuk Tung Liu, Stuart L. Shapiro, and Thomas W. Baumgarte. Relativistic Simulations of Black Hole-Neutron Star Mergers: Effects of black-hole spin. Phys. Rev., D79:044024, 2009 .

[15] Francois Foucart, Matthew D. Duez, Lawrence E. Kidder, and Saul A. Teukolsky. Black hole-neutron star mergers: effects of the orientation of the black hole spin. Phys. Rev., D83:024005, 2011.

[16] Koutarou Kyutoku, Hirotada Okawa, Masaru Shibata, and Keisuke Taniguchi. Gravitational waves from spinning black hole-neutron star binaries: dependence on black hole spins and on neutron star equations of state. Phys. Rev. D, 84:064018, 2011.

[17] Francesco Pannarale, Aaryn Tonita, and Luciano Rezzolla. Black hole-neutron star mergers and short GRBs: a relativistic toy model to estimate the mass of the torus. Astrophys.J., 727:95, 2011.

[18] Francois Foucart, Matthew D. Duez, Lawrence E. Kidder, Mark A. Scheel, Béla Szilágyi, and Saul A. Teukolsky. Black hole-neutron star mergers for $10 M_{\odot}$ black holes. Phys. Rev. D, 85(4):044015, February 2012.

[19] F. Foucart. Black-hole-neutron-star mergers: Disk mass predictions. Phys. Rev. D, 86(12):124007, December 2012.

[20] James M. Bardeen, William H. Press, and Saul A Teukolsky. Rotating black holes: Locally nonrotating frames, energy extraction, and scalar synchrotron radiation. Astrophys. J., 178:347, 1972.

[21] V. S. Safronov. On the gravitational instability in flattened systems with axial symmetry and nonuniform rotation. Annales d'Astrophysique, 23:979, February 1960.

[22] A. Toomre. On the gravitational stability of a disk of stars. Astrophys. J., 139:1217-1238, May 1964.

[23] J. Goodman and R. Narayan. The stability of accretion tori. III - The effect of self-gravity. MNRAS, 231:97-114, March 1988.

[24] D. M. Christodoulou and R. Narayan. The stability of accretion tori. IV - Fission and fragmentation of slender, self-gravitating annuli. Astrophys. J., 388:451-466, April 1992.

[25] J. W. Woodward, J. E. Tohline, and I. Hachisu. The stability of thick, self-gravitating disks in protostellar systems. Astrophys. J., 420:247-267, January 1994.

[26] N. Masuda, S. Nishida, and Y. Eriguchi. The runaway instability of self-gravitating tori with nonconstant specific angular momentum around black holes. MNRAS, 297:1139-1144, July 1998.

[27] P. A. Taylor, J. C. Miller, and P. Podsiadlowski. Long duration gamma-ray bursts: hydrodynamic instabilities in collapsar disks. MNRAS, 410:2385, June 2010.

[28] P. J. Montero, J. A. Font, and M. Shibata. Influence of Self-Gravity on the Runaway Instability of 
Black-Hole-Torus Systems. Physical Review Letters, 104(19):191101, May 2010.

[29] L. Rezzolla, L. Baiotti, B. Giacomazzo, D. Link, and J. A. Font. Accurate evolutions of unequalmass neutron-star binaries: properties of the torus and short GRB engines. Class. Quantum Grav., 27(11):114105, June 2010.

[30] O. Korobkin, E. B. Abdikamalov, E. Schnetter, N. Stergioulas, and B. Zink. Stability of generalrelativistic accretion disks. Phys. Rev. D, 83(4):043007, February 2011.

[31] K. Kiuchi, M. Shibata, P. J. Montero, and J. A. Font. Gravitational Waves from the Papaloizou-Pringle Instability in Black-Hole-Torus Systems. Phys. Rev. Lett., 106(25):251102, June 2011.

[32] O. Korobkin, E. Abdikamalov, N. Stergioulas, E. Schnetter, B. Zink, et al. The runaway instability in general-relativistic accretion disks. 2012.

[33] M. A. Abramowicz, V. Karas, and A. Lanza. On the runaway instability of relativistic tori. Astron. Astrophys., 331:1143-1146, March 1998.

[34] F. Daigne and J. A. Font. The runaway instability of thick discs around black holes - II. Non-constant angular momentum discs. MNRAS, 349:841-868, April 2004.

[35] J. C. B. Papaloizou and J. E. Pringle. The dynamical stability of differentially rotating discs. II. MNRAS, 213:799-820, April 1985.

[36] R. Popham, S. E. Woosley, and C. L. Fryer. Hyperaccreting black holes and gamma-ray bursts. Ap. J., 518:356, 1999.

[37] S. Setiawan, M. Ruffert, and H.-T. Janka. Three-dimensional simulations of non-stationary accretion by remnant black holes of compact object mergers. Astron. Astrophys., 458:553, 2006.

[38] W.-X. Chen and A. M. Beloborodov. Neutrino-cooled Accretion Disks around Spinning Black Holes. Astrophys. J., 657:383-399, March 2007.

[39] M. Shibata, Y. Sekiguchi, and R. Takahashi. Magnetohydrodynamics of Neutrino-Cooled Accretion Tori around a Rotating Black Hole in General Relativity. Prog. Theor. Phys., 118:257-302, August 2007.

[40] R. D. Blandford and R. L. Znajek. Electromagnetic extraction of energy from Kerr black holes. Mon. Not. Roy. Astr. Soc., 179:433-456, May 1977.

[41] Francois Foucart, Lawrence E. Kidder, Harald P. Pfeiffer, and Saul A. Teukolsky. Initial data for black hole-neutron star binaries: a flexible, high-accuracy spectral method. Phys. Rev. D, 77:124051, 2008.

[42] Geoffrey Lovelace, Robert Owen, Harald P. Pfeiffer, and Tony Chu. Binary-black-hole initial data with nearly-extremal spins. Phys. Rev. D, 78:084017, 2008.

[43] Harald P. Pfeiffer, Duncan A. Brown, Lawrence E. Kidder, Lee Lindblom, Geoffrey Lovelace, and Mark A. Scheel. Reducing orbital eccentricity in binary black hole simulations. Class. Quantum Grav., 24(12):S59-S81, 2007.

[44] http://www.black-holes.org/SpEC.html.

[45] Matthew D. Duez, Francois Foucart, Lawrence E. Kidder, Harald P. Pfeiffer, Mark A. Scheel, and Saul A. Teukolsky. Evolving black hole-neutron star binaries in general relativity using pseudospectral and finite difference methods. Phys. Rev. D, 78:104015, 2008.

[46] Lee Lindblom, Mark A. Scheel, Lawrence E. Kidder, Robert Owen, and Oliver Rinne. A new generalized harmonic evolution system. Class. Quantum Grav., 23:S447-S462, 2006.

[47] Xu-Dong Liu, Stanley Osher, and Tony Chan. Weighted essentially non-oscillatory schemes. Journal of Computational Physics, 115(1):200 - 212, 1994.

[48] Guang-Shan Jiang and Chi-Wang Shu. Efficient implementation of weighted eno schemes. Journal of Computational Physics, 126(1):202 - 228, 1996.

[49] B. van Leer A. Harten, P. D. Lax. On upstream differencing and godunov-type schemes for hyperbolic conservation laws. SIAM Rev., 25:35, 1983.

[50] Geoffrey Lovelace, Mark. A. Scheel, and Béla Szilágyi. Simulating merging binary black holes with nearly extremal spins. Phys. Rev. D, 83:024010, 2011.

[51] Geoffrey Lovelace, Michael Boyle, Mark A. Scheel, and Béla Szilágyi. High-accuracy gravitational waveforms for binary-black-hole mergers with nearly extremal spins. Class. Quant. Grav., 29:045003, 
2012.

[52] Daniel A. Hemberger, Mark A. Scheel, Lawrence E. Kidder, Béla Szilágyi, and Saul A. Teukolsky. Dynamical Excision Boundaries in Spectral Evolutions of Binary Black Hole Spacetimes. Class. Quantum Grav., 30(11):115001, 2013.

[53] F. H. Seguin. The stability of nonuniform rotation in relativistic stars. Astrophys. J., 197:745-765, May 1975.

[54] B. Giacomazzo, R. Perna, L. Rezzolla, E. Troja, and D. Lazzati. Compact Binary Progenitors of Short Gamma-Ray Bursts. ArXiv e-prints, October 2012.

[55] L. F. Roberts, D. Kasen, W. H. Lee, and E. Ramirez-Ruiz. Electromagnetic Transients Powered by Nuclear Decay in the Tidal Tails of Coalescing Compact Binaries. Astrophys. J. Lett., 736:L21, July 2011.

[56] K. Hotokezaka, K. Kiuchi, K. Kyutoku, H. Okawa, Y.-i. Sekiguchi, M. Shibata, and K. Taniguchi. Mass ejection from the merger of binary neutron stars. Phys. Rev. D, 87(2):024001, January 2013.

[57] Matthew D. Duez, Francois Foucart, Lawrence E. Kidder, Christian D. Ott, and Saul A. Teukolsky. Equation of state effects in black hole-neutron star mergers. Class. Quant. Grav., 27:114106, 2010.

[58] F. Foucart, M. B. Deaton, M. D. Duez, L. E. Kidder, I. MacDonald, C. D. Ott, H. P. Pfeiffer, M. A. Scheel, B. Szilagyi, and S. A. Teukolsky. Black hole-neutron star mergers at realistic mass ratios: Equation of state and spin orientation effects. Accepted for publication in Phys. Rev. D., arXiv:1212.4810, March 2013. 